\title{
Dysphagia Caused by Two Possible Culprits - Diverticulum or Osteophyte
}

\author{
Brian Walker ${ }^{1 *}$, Naresh Patel ${ }^{2}$ and William Karle ${ }^{1}$ \\ ${ }^{1}$ Department of Otolaryngology, The Mayo Clinic in Arizona, Phoenix, AZ, USA \\ ${ }^{2}$ Department of Neurosurgery, The Mayo Clinic in Arizona, Phoenix, AZ, USA \\ *Corresponding Author: Brian Walker, Department of Otolaryngology, The Mayo \\ Clinic in Arizona, Phoenix, AZ, USA.
}

Received: August 25, 2021

Published: September 11, 2021

(C) All rights are reserved by Brian Walker., et al.

\begin{abstract}
Killian-Jamieson Diverticulum (KJD) is a rare and often misdiagnosed esophageal diverticulum with a wide variability of presentation and treatment options.

We report the first case of KJD treated with cervical osteophytectomy in addition to transcervical diverticulectomy. A 76 year old male presented with a history of dysphagia with KJD and imaging findings of cervical osteophytes directly adjacent to the diverticulum. He was able to resume oral intake on post- operative day one and reported significant improvement in symptoms at follow-up.

It is therefore important to consider extra-esophageal contributions to diverticulum development. Transcervical diverticulectomy with osteophytectomy is a safe and effective method of managing KJD.
\end{abstract}

Keywords: Killian-Jamieson Diverticulum; Cervical Osteophyte; Osteophytectomy; Dysphagia; Zenker's Diverticulum

\section{Abbreviations}

KJD: Killian-Jamieson Diverticulum; CT: Computed Tomography

\section{Introduction}

Killian-Jamieson Diverticulum (KJD) is a rare and often misdiagnosed esophageal diverticulum with a wide variability of presentation and treatment options.

\section{Case Report}

A 76 year old male with a history of cervical trauma presented with dysphagia to solids and liquids with frequent food regurgitation and 15 pound weight loss over the past year. An otolaryngologist at an outside institution diagnosed the patient with a Zenker's diverticulum based on modified barium swallow and ordered him for an esophagogastroduodenoscopy which did not identify any abnormality. He then presented to our institution for a second opinion.

The patient's outside barium swallow was reviewed and demonstrated a left-sided esophageal diverticulum inferior to the cricopharyngeus, thus allowing diagnosis of a Killian-Jamieson diverticulum and not a Zenker's diverticulum (Figure 1a). Subsequent CT neck with contrast demonstrated cervical osteophytes at levels C5-T1 (Figure 1b), directly adjacent to the diverticulum. It could not be definitively determined whether the patient's dysphagia was due primarily from the osteophyte compression, the diverticulum, or an equal contribution of the two. Therefore, we elected to perform a transcervical diverticulectomy with concomitant osteophy- 
tectomy to spare the patient the need for a potential second stage procedure.

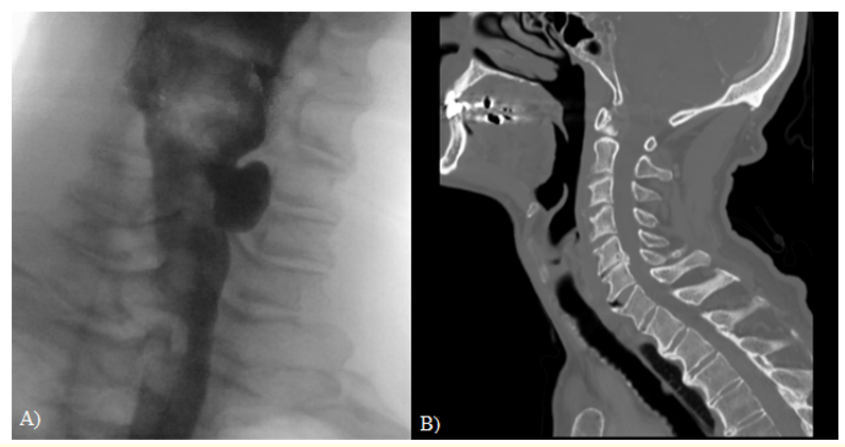

Figure 1: Pre-operative imaging demonstrating a KillianJamieson diverticulum and cervical osteophytes (C5-T1). (A) Anterior-posterior view of fluoroscopic swallow study and (B) Sagittal view of CT neck.

Intraoperatively, a standard transverse left neck incision was used and the case began with the osteophytectomy which included levels C6-C7. Following this, the diverticulum was exposed with special attention to identifying and preserving the left recurrent laryngeal nerve (Figure 2a). Intraoperative findings include significant adhesions between the diverticulum and the ipsilateral thyroid gland. The diverticulum was then removed using a stapler and measured $2 \mathrm{~cm} \times 1.5 \mathrm{~cm}$ (Figure 2b). Postoperatively the patient was made NPO and on POD \#1 gastrograffin esophagram did not demonstrate any extravasation or persistence of diverticula. The patient was discharged home with instructions to advance his diet over the following two weeks. At his one month post-op visit he reported complete resolution of his dysphagia and had clinically significant improvements as measured by both the MD Anderson Dysphagia Inventory and EAT 10 scores which improved by 18 (53 pre, 71 post) and 23 ( 25 pre, 2 post) points, respectively.

\section{Discussion}

KJD are rare lateral esophageal diverticula which form at the juncture of the lateral wall of the longitudinal muscle of the esophagus and the inferior border of the cricopharyngeus [1,2]. These diverticula are commonly misdiagnosed as a Zenker's diverticulum,

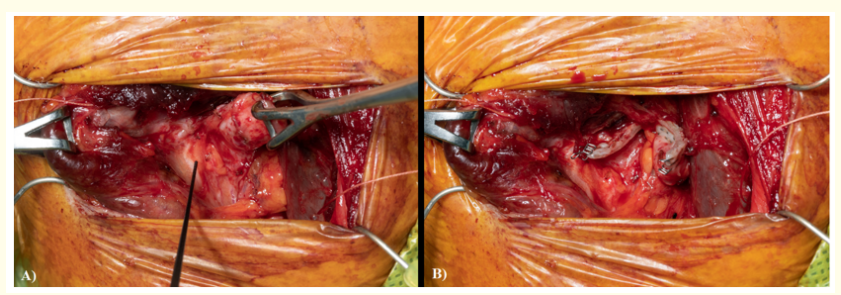

Figure 2: Intraoperative appearance of Killian-Jamieson diverticulum. (A) identification of the left recurrent laryngeal nerve, (B) after stapling.

as was the case with our patient [3,4]. Although these diverticula are often asymptomatic, patients may present with dysphagia, regurgitation, emesis, choking, and globus sensation [5]. Both open and endoscopic treatment has been advocated in prior reports [6-8]. We report successful treatment, as measured by subjective criteria, of our patient's KJD via transcervical diverticulectomy with osteophytectomy at levels C6-C7, without CP myotomy and without complication or significant delay in the patient's return to swallow.

Unlike previous reports in the literature, our patient was found to have cervical osteophytes at the same level of the KJD, which may have contributed either directly or indirectly to his dysphagia and the development of the KJD. Prior reports in the literature describe dysphagia secondary to compression of the esophagus due to impingement from anterior cervical osteophytes. Additionally, it is believed that osteophytes can induce a localized inflammatory reaction causing scarring of the esophagus resulting in dysphagia, which is consistent with our findings of significant adhesions involving our patient's KJD [9]. While it is impossible to determine the specific etiology of our patient's KJD, his symptoms did resolve with management of both the KJD and the cervical osteophytes.

Despite undergoing osteophytectomy in addition to transcervical diverticulectomy, our patient was able to resume a PO diet less than 24 hours after surgery with immediate improvement in his symptoms. This was considerably faster than the average of four days reported in the literature for transcervical approaches [5]. 
To our knowledge, there have been no reports of subjective outcome measures among patients who have undergone KJD excision. Our patient had clinically significant improvements as measured by both the MD Anderson Dysphagia Inventory and EAT 10 scores [10].

\section{Conclusion}

$\mathrm{KJD}$ is a rare and often misdiagnosed esophageal diverticulum with a wide variability of presentation and treatment options. We report the first case of KJD treated with cervical osteophytectomy in addition to transcervical diverticulectomy. When evaluating KJD it is important to consider extra-esophageal contributions to diverticulum development such as cervical osteophyte compression of the esophagus. Special attention should be paid to the recurrent laryngeal nerve due to its close proximity to the diverticulum, especially within the context of potential scarring and adhesions surrounding the KJD. Transcervical diverticulectomy with osteophytectomy is a safe and effective method of managing KJD.

\section{Bibliography}

1. Eckberg $\mathrm{O}$ and Nylander G. "Lateral diverticula from the pharyngo-esophageal junction area". Radiology 146 (1983): 117122.

2. Saisho K., et al. "Surgery for Killian-Jamieson diverticulum: a report of two cases". Surgical Case Reports 6 (2020): 17.

3. Rodgers PJ., et al. "Killian-Jamieson diverticulum: a case report and a review of the literature". Annals of Otology, Rhinology and Laryngology: SAGE Journals 109 (2000): 1087-1091.

4. Rubesin SE and Levine MS. "Killian-Jamieson diverticula: radiographic findings in 16 patients". AJR 177 (2001): 85-89.

5. Haddad N., et al. "Presentation and management of Killian-Jamieson diverticulum: a comprehensive literature review". Annals of Otology, Rhinology and Laryngology: SAGE Journals 129.4 (2020): 394-400.

6. Kim DC., et al. "Surgical treatment of Killian-Jamieson Diverticulum". The Korean Journal of Thoracic and Cardiovascular Surgery 45 (2012): 272-274.

7. Sanders D., et al. "Endoscopic management of Killian-Jamieson diverticulum". Video GIE 4.8 (2019): 364-365.
8. Siow SL., et al. "Transcervical diverticulectomy for Killian-Jamieson diverticulum". Asian Journal of Surgery 40 (2017): 324328.

9. Carucci LR and Turner MA. "Dysphagia revisited: common and unusual causes". Radiographics 35 (2015): 105-122.

10. Hutcheson KA., et al. "What is a clinically relevant difference in MDADI scores between groups of head and neck cancer patients?" Laryngoscope 126.5 (2016): 1108-1113.

\section{Volume 3 Issue 10 October 2021 \\ (C) All rights are reserved by Brian Walker., et al.}

